\title{
The temporal structure of professional speaking styles in Brazilian Portuguese
}

\author{
Luciana Castro $^{1}$ and João Antônio de Moraes ${ }^{1,2}$ \\ ${ }^{1}$ Laboratory of Acoustic Phonetics, Universidade Federal do Rio de Janeiro, Brazil \\ ${ }^{2}$ Conselho Nacional de Desenvolvimento Científico e Tecnológico (CNPq) \\ https://doi.org/10.36505/ExLing-2008/02/0015/000074
}

\begin{abstract}
This study describes the temporal organization of speech collected from politicians, religious leaders and television news anchors, individuals for whom speech represents an essential component of their professional identity. Analysis of duration measurements of the recorded speech suggests that the temporal differences observed between the speaking styles are statistically significant.
\end{abstract}

Key words: prosody, speaking style, temporal organization of speech

\section{Introduction}

The relation between prosody and the characterization of a professional speaking style is noted by Léon (1993), who observes that different situations (public speaking, reading, theater, etc.) result in different speaking styles which over time become permanently associated with particular professions. This study investigates whether the temporal structure of speech differs significantly among the professional speaking styles of the politician, the religious leader, and the news broadcaster for Brazilian Portuguese.

\section{Method}

The analysis is based on the professional speech of six adult speakers: two presenters of TV news, two TV ministers (religious leaders), and two politicians. The speakers, ranging in age from 35 to 55 , are native speakers of Brazilian Portuguese with no apparent speaking disorders.

Each recording consisted of two minutes of speech captured during the exercise of each speaker's profession: the TV news presenters in the studio, the politicians in the senate, and the religious leaders in their studio/church. All of the recordings were captured directly from Brazilian broadcast television. From observing the video, it was apparent that none of the speakers was reading directly from a text, though it is assumed that the TV news anchors were working with the support of a teleprompting machine.

The recordings, made on a laptop computer connected to the audio output of an analog television, were digitized using a sampling rate of $22 \mathrm{kHz}$ and analyzed using the acoustic analysis software Speech Analyzer.

ExLing 2008: Proceedings of 2nd Tutorial and Research Workshop on Experimental Linguistics, 25-27 August 2008, Athens, Greece 
The recordings were transcribed orthographically, and then silent and filled pauses in the speech were identified through a combination of perceptive analysis (listening) and the visual inspection of the spectrograms. As a rule, any interruption of more than 40 milliseconds in the flow of speech was considered a pause. The result of this process was the segmentation of the speech in two units: pauses and speech sequences.

Based on this segmentation, it was possible to derive the following metrics: the total time of the pauses (TP), the number of pauses (NP), the average pause duration (AP), the pause time per syllable (TP/syl), the speech rate (SR), and the articulation rate (AR).

\section{Results and discussion}

\section{Types of pauses: silent $v$ s. filled}

Filled pauses are completely absent in the TV news speaking style, whereas in the sermon and in the political speech, 3.9\% and $10.6 \%$ of the pauses, respectively, are filled pauses, suggesting a greater degree of spontaneity in the speech of the politician as compared to the other professional speaking styles.

\section{Number and duration of pauses}

The TV news speaking style exhibited the fewest number of pauses, followed by the political and religious styles, as can be seen in Figure 1.

The average pause duration, in principle independent of the number of pauses, follows exactly the same order, with the TV news speaking style having the shortest average pause duration, followed by the political speaking style, and finally the religious speaking style (Figure 1). As such, the total pause time (TP, not shown here), being the product of the number of pauses and the average pause time, exhibits the same tendencies (to a greater degree) present in each of the component variables.

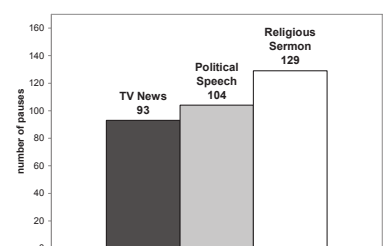

A

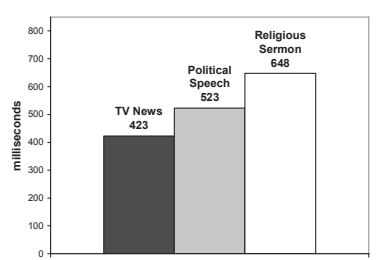

B

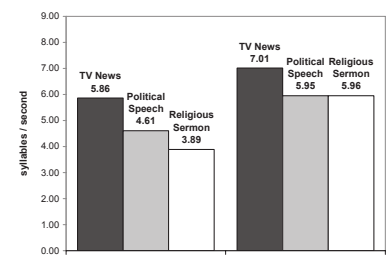

$\mathrm{C}$

Figure 1: Total number of pauses (A), average pause duration (B), and speech and articulation rate $(\mathrm{C})$ for each speaking style.

Previous studies (Sabin et al. 1979) have observed that read speech employs fewer pauses than spontaneous speech, which may explain the 
lower number of pauses observed here in the speech of the TV news broadcasters. Similarly, Goldman-Eisler (1968) reports that the total pause duration [TP] for "descriptive" speech is half that of "interpretive" speech. One factor that may contribute to this observed difference in the speech of religious leaders and politicians is their use of pauses for dramatic effect (Duez, 1991).

\section{Speaking rate}

Figure 1 (above) also presents the results of measuring both the overall speech rate and the articulation rate, which considers only the segments of the recording labeled as "speech sequences" (that is, excluding the pauses).

The religious and political speaking styles were delivered at the same articulation rate (5.96 syllables/second), while the TV news was presented $17.6 \%$ faster $(7.01 \mathrm{syl} / \mathrm{sec})$. When the pauses are taken into account, overall speech rate inversely reflects the tendencies previously reported for total pause time, with the religious speaking style presenting the slowest speech rate at $3.89 \mathrm{syl} / \mathrm{sec}$, followed by the political speaking style at $4.61 \mathrm{syl} / \mathrm{sec}$ and, finally, the TV news speaking style with the fastest speech rate at 5.86 syl/sec. Shevchenko and Uglova (2006) observed speaking rates of 5.1 syl/sec for TV news. Delgado-Martins and Freitas (1991) suggested that the speech of TV news broadcasters may be faster due in part to the severe time restrictions imposed on the delivery of the news, a factor that may also explain the reduced average duration of the pauses.

\section{Statistical analysis}

The histograms in Figure 2 suggest that the pause durations of each speaking style do not obey a normal distribution. In order to evaluate the significance of the differences observed between the speaking styles, the non-parametric Kruskal-Wallis test, which does not assume a normal distribution, was applied. The test was applied using the statistical software package " $R$ " and resulted in a p-value of $2.294 \times 10-6$ - that is, significant for $\alpha=0.01$.

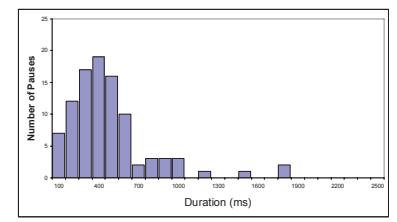

TV News

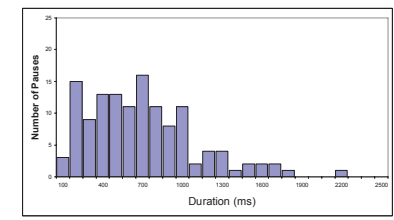

Religious Speech

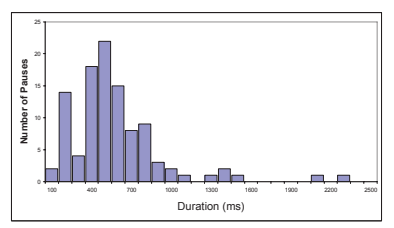

Political Speech

Figure 2: Histograms of pause durations for each speaking style.

Note that the religious speaking style, in addition to being the style with the highest pause duration, is also the style with the largest variance. 


\section{Conclusions}

The temporal characteristics observed in this study corroborate the findings reported in previous work in several regards. First, the semi-spontaneous speech of the religious leaders and politicians was characterized by a greater number and average duration of pauses and by a slower speaking rate than that of the TV news broadcasters. These results are in accordance with the idea that, in speech in which lexical and syntactic choices are the responsibility of the speaker in real time, the time spent in pauses suffers an increase (Sabin et al. 1979). Furthermore, a total absence of filled pauses was observed in the TV news speaking style, in contrast to the political and religious speaking styles.

A statistical analysis of the data revealed significant differences in the pause duration distributions of the three speaking styles studied here. However, due to the limited size of the corpus, it was not possible to infer a direct relation between the professional speaking style per se and the observed values. Specifically, it was not possible to reject the hypothesis that the differences observed are a result of the personal speaking style of each speaker, as opposed to the professional speaking style.

\section{References}

Delgado-Martins, M.R. and Freitas, M.J. 1991. Temporal structures of speech: reading news on TV. Proceedings of the ESCA Workshop, Barcelona, Spain.

Duez, D. 1991. La pause dans la parole de l'homme politique. Paris, CNRS.

Goldman-Eisler, F. 1968. Psycholinguistics: experiments in spontaneous speech. London and New York, Academic Press.

Léon, P. 1993. Variation situationnelle et voix professionnelles. In: Précis de Phonostylistique. Paris, Nathan.

Sabin, E; Clemmer, E; O Connell, D; Kowal, S. 1979. A pausological approach to speech development. In: Siegman, A. and Feldstein, S. (eds.) Of speech and time. New Jersey, LEA Publishers.

Shevchenko, T and Uglova, N. 2006. Timing in news and weather forecasts: implications for perception. Proceedings of Speech Prosody, Dresden, Germany. 